The University of San Francisco

USF Scholarship: a digital repository @ Gleeson Library |

Geschke Center

1997

\title{
Constraints on the Production of Ultra-High- Energy Cosmic Rays by Isolated Neutron Stars
}

Aparna Venkatesan

University of San Francisco, avenkatesan@usfca.edu

M Coleman Miller

Angela V. Olinto

Follow this and additional works at: http://repository.usfca.edu/phys

Part of the Astrophysics and Astronomy Commons, and the Physics Commons

\section{Recommended Citation}

Aparna Venkatesan, M. Coleman Miller and Angela V. Olinto. Constraints on the Production of Ultra-High-Energy Cosmic Rays by Isolated Neutron Stars. The Astrophysical Journal, 484:323-328, 1997 July 20 http://dx.doi.org/10.1086/304304

This Article is brought to you for free and open access by the College of Arts and Sciences at USF Scholarship: a digital repository @ Gleeson Library | Geschke Center. It has been accepted for inclusion in Physics and Astronomy by an authorized administrator of USF Scholarship: a digital repository @ 
ThE Astrophysical Journal, 484:323-328, 1997 July 20

(C) 1997. The American Astronomical Society. All rights reserved. Printed in U.S.A.

\title{
CONSTRAINTS ON THE PRODUCTION OF ULTRA-HIGH-ENERGY COSMIC RAYS BY ISOLATED NEUTRON STARS
}

\author{
Aparna Venkatesan, M. Coleman Miller, ${ }^{1}$ and Angela V. Olinto \\ Department of Astronomy and Astrophysics, and Enrico Fermi Institute, University of Chicago, \\ 5640 South Ellis Avenue, Chicago, IL 60637 \\ Received 1996 July 5 ; accepted 1997 February 10
}

\begin{abstract}
The energetics, spectrum, and composition of cosmic rays with energies below $\sim 10^{15} \mathrm{eV}$ are fairly well explained by models involving supernova shocks. In contrast, no widely accepted theory exists for the origin of ultra-high-energy cosmic rays (UHECRs), which have energies above $10^{15} \mathrm{eV}$. Instead of proposing a specific model, here we place strong constraints on any model of UHECRs involving isolated neutron stars (no companions). We consider the total power requirements and show that the only viable power source associated with isolated neutron stars is rotation. Mechanisms based on accretion from the interstellar medium fall short of the necessary power despite the most optimistic assumptions. Power considerations also demonstrate that not enough rotational energy is tapped by a "propeller"like acceleration of interstellar matter. The most promising source of energy is rotational spindown via magnetic braking. We examine microphysical energy loss processes near magnetized neutron stars and conclude that the most likely site for yielding UHECRs from isolated neutron stars is near or beyond the light cylinder.
\end{abstract}

Subject headings: acceleration of particles - cosmic rays - stars: neutron

\section{INTRODUCTION}

The energy spectrum of cosmic rays is well established between $\sim 10^{8}$ and $\sim 10^{20} \mathrm{eV}$ (Axford 1994; Bird et al. 1994, and references therein). There is clearly a "knee" at about $10^{15} \mathrm{eV}$, at which the spectrum changes from $N(E) \sim E^{-2.7}$ to $N(E) \sim E^{-3.1}$, and an " ankle" centered on $10^{18.5} \mathrm{eV}$, beyond which $N(E) \sim E^{-2.7}$. Cosmic rays of energy up to the knee are widely accepted as originating in shocks associated with galactic supernova remnants, but supernova shocks have difficulties producing particles of higher energy. It is therefore necessary to posit another process to produce these ultra-high-energy cosmic rays (UHECRs) beyond the knee of the spectrum. Cosmic rays with energies above $\sim 10^{19} \mathrm{eV}$ are generally thought to be extragalactic (Axford 1994; Bird et al. 1994), although they may also originate in an extended halo of the Galaxy (Vietri 1996).

Recent attention has focused on isolated neutron stars (without any companions) as promising sites for highenergy phenomena, such as X-ray and UV radiation (Blaes \& Madau 1993; Madau \& Blaes 1994, hereafter BM93 and MB94, respectively), as well as ultra-high-energy gamma rays (see, e.g., Harding 1990). In particular, it has been suggested that accretion from the interstellar medium by isolated neutron stars may provide the necessary energetics and spectrum up to cosmic-ray energies of $\sim 10^{15} \mathrm{eV}$ (Shemi 1995). Here we analyze in detail the prospects for these isolated neutron stars to be the source of cosmic rays above $10^{15} \mathrm{eV}$. We narrow down the set of allowed models by requiring first that any model be able to generate the total power observed in UHECRs, then by examining microphysical energy loss processes to determine the maximum energy to which cosmic rays may be accelerated around neutron stars.

The plan of this paper is as follows. In $\S 2$ we review the data on the energy and spectrum of cosmic rays above the

\footnotetext{
${ }^{1}$ Compton GRO Fellow.
}

knee. The energy generation rate of cosmic rays depends on both the observed spectrum and the dependence of confinement time on energy. The latter is uncertain past $\sim 10^{12} \mathrm{eV}$ (Müller et al. 1991), but we show that at least $10^{38} \mathrm{ergs} \mathrm{s}^{-1}$ of cosmic rays beyond $10^{15} \mathrm{eV}$ leave the Galaxy. This is thus the energy generation rate of any viable mechanism. In $\S 3$ we consider specific power sources associated with isolated neutron stars, including magnetic fields, kinetic energy, accretion from the interstellar medium, and rotation. We find that only rotation produces the required power per neutron star; in particular, accretion from the interstellar medium is too weak. We show further that a "propeller" mechanism (Illarionov \& Sunyaev 1975) is not likely to convert rotational energy to UHECRs, because ram pressure from the neutron star wind prevents accretion until the rotational energy is too low to account for UHECRs. If rotational energy is transformed into UHECRS, it is likely to do so directly from the neutron star wind. In $\S 4$ we consider microphysical loss processes near magnetized neutron stars to determine the maximum energy to which a particle may be accelerated. We find that synchrotron and curvature radiation are the most significant loss processes but that if acceleration takes place near the light cylinder the losses may be negligible. We also show that the preferential escape of higher energy particles may occur beyond the light cylinder. Finally, in $\S 5$ we discuss our results and summarize the viable mechanisms for the production of UHECRs by isolated neutron stars.

\section{POWER AND SPECTRAL REQUIREMENTS}

The energy generation rate of cosmic rays in the Galaxy above a given energy $E_{0}$ is

$$
P\left(\geq E_{0}\right) \propto \int_{E_{0}}^{\infty} N(E) E E^{\eta} d E .
$$

Here $N(E)$ is the differential number distribution of observed cosmic rays, and the factor $E^{\eta}$ accounts for the 


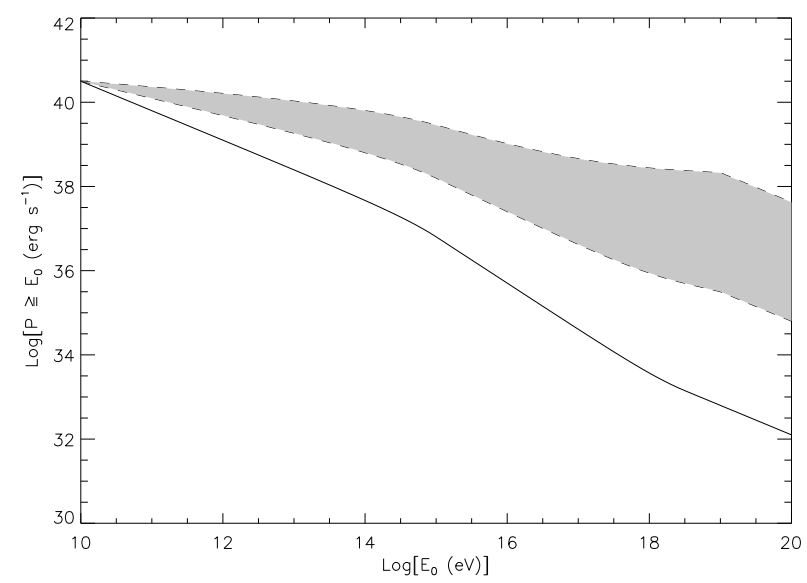

FIG. 1.-Power requirements as a function of the energy of cosmic rays. Shaded area is bounded from below by $\eta=0.3$ and from above by $\eta=0.6$. Solid line is for $\eta=0$.

dependence of the galactic confinement time on the cosmic rays' energy. Empirically, $N(E) \propto E^{-\gamma}$, with a spectral index $\gamma=2.7$ for $E \lesssim 10^{15} \mathrm{eV}$ and $\gamma=3.1$ for $10^{15} \mathrm{eV} \lesssim E \lesssim$ $10^{18.5} \mathrm{eV}$. There is evidence that the spectrum hardens again to $\gamma \sim 2.7$ above $\sim 10^{18.5} \mathrm{eV}$ (Bird et al. 1994, and references therein).

Cosmic rays of higher energy have larger gyroradii, and thus escape more easily from the Galaxy, than cosmic rays of lower energy. Above $\sim 10^{19} \mathrm{eV}$ cosmic rays are no longer confined by the galactic magnetic field, so $\eta \rightarrow 0$; below this energy the original source spectrum of cosmic rays will be flattened. To understand this effect, consider that the power necessary to explain the observed luminosity in cosmic rays with energy above $E_{0}$ is $P\left(\geq E_{0}\right) \propto \tau_{\text {conf }}^{-1}$ (Milgrom \& Usov 1996), where $\tau_{\text {conf }}$ is the confinement time. We assume here that $\tau_{\text {conf }}$ depends on energy as $E^{-\eta}$. On theoretical grounds, Biermann (1993) estimated $\eta \sim 0.3$ for the energy range $10^{13}$ to $3 \times 10^{18} \mathrm{eV}$. However, from measurements of the relative abundance of secondaries in the cosmic ray spectrum and the predicted escape rates at a given energy Müller et al. (1991) determined that $\eta \simeq 0.6$ between $10^{10}$ and $10^{12} \mathrm{eV}$. Outside this energy range, there are no empirical determinations of $\eta$.

In Figure 1, we plot the power requirement as a function of energy from $10^{10}$ to $10^{20} \mathrm{eV}$, taking the uncertainty in $\eta$ into account. The shaded region of the graph represents the range in the power for possible values of $\eta$, with the lower bound drawn for $\eta=0.3$ and the upper bound for $\eta=0.6$. The solid curve has $\eta=0$; confinement effects are also neglected for $E_{0}>10^{19} \mathrm{eV}$. Each curve is normalized by setting $P\left(\geq 10^{10} \mathrm{eV}\right)=10^{40.5} \mathrm{ergs} \mathrm{s}^{-1}$ (Milgrom \& Usov 1996). From this curve we find that the minimum energy generation rate in the Galaxy past $10^{15} \mathrm{eV}$ is $\sim 10^{38}$ ergs $\mathrm{s}^{-1}$, and so this is the minimum power that must be met by models seeking to explain UHECRs.

\section{POWER SOURCES}

Since neutron stars are produced in supernovae at a maximum rate of one per ten years, or one every $10^{8.5} \mathrm{~s}$, they have to produce at least $10^{38} \times 10^{8.5}=10^{46.5}$ ergs per neutron star in UHECRs during their lifetimes in order to account for cosmic rays above the knee.

Neutron stars have many sources of energy, but some of them are not promising as sources of cosmic rays. For example, if their average magnetic field is $\lesssim 10^{13} \mathrm{G}$, as is inferred for all rotationally powered pulsars, then each neutron star has a total magnetic energy $\lesssim 10^{43} \mathrm{ergs}$, many orders of magnitude too low. Observations of pulsar birth velocities (see below) tell us that the kinetic energy of neutron stars could easily account for cosmic rays; at a median space velocity of $500 \mathrm{~km} \mathrm{~s}^{-1}$ the energy per neutron star is $\approx 4 \times 10^{48}$ ergs. To tap that energy the star must be slowed down by collisions. However, collisions between stars are extremely improbable in the disk of the Galaxy, and typical interstellar medium (ISM) densities are not sufficient to slow down neutron stars over the age of the universe.

We are therefore left with two main energy sources, which we consider below: accretion and rotation. In this paper we consider only isolated neutron stars, so by the accretion we mean accretion from the ISM. Although accretion has been proposed as a promising source of cosmic rays, we find that the power that can be generated is insufficient to account for UHECRs, let alone all cosmic rays of energy above $10^{10}$ $\mathrm{eV}$. Therefore, rotation is the only viable power source.

\subsection{Accretion from the ISM}

Recent work has suggested that neutron stars accreting from the ISM may be sources of UV and X-ray radiation (BM93; MB94). Typically, Bondi-Hoyle accretion (Bondi \& Hoyle 1944) is considered, which becomes much less efficient with increasing neutron star velocities. Our understanding of the velocity distribution of neutron stars has been revised significantly in the last few years; prior to 1993, their average velocity was assumed to be $\sim 150 \mathrm{~km} \mathrm{~s}^{-1}$. However, Lyne \& Lorimer (1994) showed that after selection effects were removed, this number increased to $\sim 450$ $\mathrm{km} \mathrm{s}^{-1}$. Frail, Goss, \& Whiteoak (1994) inferred that neutron stars associated with supernova remnants have average velocities of $\sim 500 \mathrm{~km} \mathrm{~s}^{-1}$. These results greatly lessen the overall power from ISM-accreting neutron stars, as compared to earlier estimates.

Bondi-Hoyle accretion is the most efficient accretion possible onto the star from the ISM and, therefore, gives an upper limit to the luminosity. A neutron star in the disk of the Galaxy with a spatial velocity of $200 v_{200} \mathrm{~km} \mathrm{~s}^{-1}$, moving through an ISM of average number density $n_{0}$ $\mathrm{cm}^{-3}$ has a Bondi-Hoyle accretion rate of $\dot{M}_{\mathrm{BH}}=3.6 \times 10^{8}$ $n_{0} v_{200}^{-3} \mathrm{~g} \mathrm{~s}^{-1}$. The energy released by this accretion cannot exceed the free-fall energy on the stellar surface, which gives $\sim 2 \times 10^{20}$ ergs $\mathrm{g}^{-1}$, or a luminosity of $L_{\mathrm{ff}} \approx 6.8 \times 10^{28}$ $n_{0} v_{200}^{-3} \mathrm{ergs} \mathrm{s}^{-1}$. An average neutron star, accreting for the entire lifetime of our Galaxy $\left(3 \times 10^{17} \mathrm{~s}\right)$, will therefore produce only $\sim 2 \times 10^{46}$ ergs via accretion from the ISM, taking $n_{0} \sim v_{200} \sim 1$. Despite the most optimistic assumptions, this is still too small to explain the observed power in UHECRs.

A realistic estimate of the accretion power will yield much less than $2 \times 10^{46} \mathrm{ergs}$, if for example, centrifugal barriers and ram pressure of the neutron star wind are taken into account (BM93, and references therein). Moreover, the efficiency of converting accretion energy into cosmic rays is certainly much less than unity, and the preheating of the ISM by radiation from the neutron star can also decrease the accretion rate (Blaes, Warren, \& Madau 1995). The total mass accreted by all isolated neutron stars in the Galaxy depends strongly on the number of low-velocity neutron stars; since the accretion rate goes as the inverse cube of the 
neutron star's velocity, for which we have assumed a lower value than the median one, our upper limit is robust.

An individual neutron star moving slowly in a very dense environment, e.g., in the cores of giant molecular clouds, could perhaps, produce cosmic rays at a high rate (Shemi 1995). However, since the accretion rate goes linearly with the ISM density, the total accretion power onto neutron stars is proportional to the average ISM density. Thus, neutron stars in dense clouds cannot be considered as typical and the overall energy constraint for UHECRs will not be met by accretion models for isolated neutron stars.

\subsection{Rotation of Neutron Stars}

The most rapidly rotating neutron star known is PSR $1937+214$, which has an angular velocity of $\sim 4 \times 10^{3} \mathrm{~s}^{-1}$ (Becker \& Helfand 1983). Since neutron stars have moments of inertia $I \sim 10^{45} \mathrm{ergs} \mathrm{s}^{2}$, a star such as PSR $1937+21$ has a rotational energy $E=\frac{1}{2} I \omega^{2} \sim 10^{52}$ ergs, many orders of magnitude greater than the $\sim 10^{46.5}$ ergs required per neutron star. Thus, even if most neutron stars rotate more slowly and the efficiency of cosmic-ray generation is much less than unity, rotation is by far the most promising cosmic-ray power source related to isolated neutron stars.

This rotational energy may be converted to the kinetic energy of particles, either from the interstellar medium or from the neutron star itself. In the first case, although the particles are accreted from the ISM and may be accelerated by, e.g., a propeller mechanism, we are not using power from accretion itself, so the constraints of the previous section do not apply. The accreted ISM may gain energy from the rotating magnetosphere through a single encounter, producing very high energy particles, or through shock acceleration involving many scatterings, leading to a powerlaw spectrum. A potential site for this is the light cylinder radius, which is the maximum cylindrical radius out to which corotation with the neutron star may be causally enforced. It is given by $r_{L}=c P / 2 \pi=4.8 \times 10^{9} P \mathrm{~cm}$, where $P$ is the period of the neutron star in seconds. Within $r_{L}$, we take the neutron star's magnetic field to have a dipole geometry $\left(B \sim r^{-3}\right)$, while beyond $r_{L}$ the magnetic field is azimuthal and has a $r^{-1}$ dependence.

However, accretion from the ISM is possible only if it is not prevented by the pressure of the neutron star wind. A rough criterion for this can be derived by equating the wind pressure, $\dot{E} / 4 \pi r^{2} c$, with the ram pressure of the infalling material, $\rho_{\text {ISM }} v^{2}$, at a radius $r$ equal to the Bondi-Hoyle radius $r_{\mathrm{BH}}=2 G M / v^{2}$. Here $\rho_{\text {ISM }}$ is the density of the ISM and $v$ is the velocity of the neutron star. The rate of rotational spin-down energy is

$$
|\dot{E}|=\frac{B^{2} R^{6} \omega^{4} \sin ^{2} \alpha}{6 c^{3}},
$$

where $B$ is the surface magnetic field of the star, $R$ is the radius of the star, $\omega$ is the star's angular velocity, and $\alpha$ is the angle between the star's magnetic and rotational axes (see, e.g., Shapiro \& Teukolsky 1983). This emitted dipole radiation is manifested as an outward wind of charged particles. We find that the ram pressure exceeds the neutron star wind pressure, and accretion occurs, only if the neutron star has spun down to a period larger than about $\sim 14.5 \mathrm{~s}$. This is consistent with the estimate of BM93 that $P_{B} \gtrsim$ $20 B_{12}^{1 / 2} n_{0}^{-1 / 4} v_{200}^{1 / 2} \mathrm{~s}$ in order for accretion to proceed, where $B=10^{12} B_{12} \mathrm{G}$, and with the estimates of other authors (see, e.g., Shemi 1995 or Harding 1990).
We apply this condition to rotation-powered neutron star models for UHECRs, where at least $10^{46.5}$ ergs are needed per neutron star. Setting this number equal to $E_{\text {rot }}=\frac{1}{2} I \omega^{2} \sim 10^{45} \omega^{2}$, we find that:

$$
P_{\max } \simeq 1.12 \mathrm{~s} \text { or } \omega_{\min } \simeq 5.6 \mathrm{~s}^{-1} .
$$

This criterion must be met if we want to generate cosmic rays of energy above $10^{15} \mathrm{eV}$ using the rotational energy of neutron stars in the Galaxy. This is incompatible with the earlier result for the minimum period necessary to allow accretion onto the neutron star. Thus, by the time that accretion can overwhelm the neutron star wind, the neutron star's rotational energy will be insufficient to account for the observed power in UHECRs, if $B \gtrsim 10^{9} \mathrm{G}$.

These difficulties can be avoided in models where particles originate from within the star's light cylinder. The conclusion of this section is therefore that, on energetic considerations alone, the only way for isolated neutron stars to be the primary source of power for UHECRs is if that power is the star's rotation. Moreover, the accelerated particles cannot come from the ISM.

\section{COMPOSITION, ACCELERATION, AND LOSS PROCESSES}

We have shown that, in viable neutron star models for UHECRs, the particles originate from within the light cylinder, and rotation is the source of power. We next examine whether these models can give the observed composition of cosmic rays and accelerate particles to the requisite energies, and if microphysical energy loss processes produce strong cutoffs in the particles' spectrum. After addressing some issues related to composition, we consider specifically an acceleration mechanism that has often been suggested as promising and involves using the potential associated with the extremely high surface fields of neutron stars. We then examine energy losses, particularly those from synchrotron and curvature emission, and, using these results, we conclude by pointing out potentially viable sites for cosmic-ray acceleration.

\subsection{Composition}

At energies of about $10^{15} \mathrm{eV}$, the spectrum of cosmic rays becomes steeper, and their composition is believed to shift from being primarily protons below this energy to mostly heavier nuclei, such as iron, above it (Bird et al. 1994; Gaisser et al. 1993). There is also some evidence for a proton component to the spectrum emerging above $10^{19} \mathrm{eV}$ (Bird et al. 1994, and references therein). A model for the origin of cosmic rays with energies in excess of $10^{15} \mathrm{eV}$ must, therefore, at least allow the composition to be biased towards heavy nuclei up to $10^{19} \mathrm{eV}$.

The ions in a neutron star wind are most likely to come from the surface of the star. This composition is difficult to predict, but it will probably be biased in the direction of iron. Simulations of fallback from Type II supernovae (which can produce neutron stars) show that the division between matter that escapes to infinity and matter that falls onto the central star occurs roughly in the silicon layer (see, e.g., Timmes, Woosley, \& Weaver 1996). If the matter accretes with a fair fraction of its free-fall velocity, it should spall into lighter elements, probably hydrogen and helium (Bildsten, Salpeter, \& Wasserman 1992). However, for much of the fallback the temperature on the surface is at least 
several hundred million degrees, implying that fusion to iron will be rapid. If accretion from the ISM is suppressed because of the neutron star wind, the propeller mechanism, or other reasons, then the composition of the atmosphere should depend on the evolution of the material accreted by fallback. Since models of cooling neutron stars indicate that the surface temperature remains above $\sim 10^{7} \mathrm{~K}$ for a few years, the light elements may be fused. Thus, although the composition of the neutron star atmosphere is by no means certain, it is plausible that it consists mainly of iron.

\subsection{Accretion by $E \| B$}

A frequently suggested mechanism for the production of high-energy ions near neutron stars is the acceleration of those ions through potential drops associated with strong electric fields parallel to the neutron star magnetic field, either near the star or farther out, near an "outer gap" region (Cheng, Ho \& Ruderman 1986). In principle, the largest potential drop associated with a neutron star with magnetic field $B$, angular velocity $\Omega$, and radius $R$ is

$$
\Phi_{\max }=300 \frac{\Omega R}{c} B R \mathrm{~V} ;
$$

so, for $\Omega=10^{4} \mathrm{~s}^{-1}, R=10^{6} \mathrm{~cm}$, and $B=10^{13} \mathrm{G}$ the maximum drop is a promisingly high $10^{21} \mathrm{~V}$. However, as in models of pulsar radio emission, the true $\Phi_{\max }$ is probably much less than $10^{21} \mathrm{~V}$. The basic problem is that if the acceleration takes place along magnetic field lines with finite radii of curvature, a seed electron accelerated by the drop will emit curvature radiation. If the curvature radiation photons are energetic enough, then when they acquire a sufficiently large angle to the magnetic field, they will produce electron-positron pairs. Each member of a pair is then accelerated but in opposite directions, and a pair cascade is formed which rapidly shuts off the potential drop. In both the polar cap model (where the acceleration is at the stellar surface; see, e.g., Ruderman \& Sutherland 1975) and the outer gap model (where the acceleration takes place at a distance of a few hundred stellar radii), the critical potential drop appears to be a comparatively paltry $\sim 10^{12}-10^{13} \mathrm{~V}$. The critical drop rises with increasing radius of curvature, but this requires a small area of emission. It is thus likely that the potential drop does not reach the required values.

Moreover, iron nuclei might not be accelerated to significantly greater energies than protons are. Binding energies in a strong magnetic field are significantly greater than they are in zero field (see, e.g., Rösner et al. 1984; Miller \& Neuhauser 1991); in a $10^{12} \mathrm{G}$ field at temperatures of $\sim 10^{6} \mathrm{~K}$, iron atoms are only partially ionized (about 3-4 times). Their energy after going through the potential drop would therefore be only 3-4, and not 26 , times that of protons, making acceleration of iron nuclei to very high energies compared to protons difficult, unless the electric field itself fully ionized the atoms.

\subsection{Energy Loss Processes}

Energetic particles moving near a neutron star experience various energy losses that influence their propagation and emergent energies. When neutron stars are young, interactions of ions with photons may be important, but for most of the life of a neutron star we expect magnetic losses to dominate.
Young neutron stars are copious emitters of thermal $\mathrm{X}$-ray photons. If the surrounding photon density is large enough, then cosmic rays accelerated near the surface will lose energy as they move through this thermal bath of photons, through processes such as inverse Compton scattering, photopion production, and photodissociation. These losses are significant for surface temperatures in excess of $\sim 10^{7} \mathrm{~K}$, implying that they may hinder the production of high-energy cosmic rays near neutron stars for a few years after the initial supernova. Past this time, energy losses to these thermal photons are minimal, and loss processes related to the stellar magnetic field dominate.

Synchrotron radiation is the fundamental process to consider when evaluating the effect of the magnetic field. Electrons of virtually any energy are constrained to follow field lines near a neutron star, but because the synchrotron energy loss rate for a given particle energy scales with the mass as $P_{\text {synch }} \sim m^{-4}$, protons or heavier ions may not follow field lines, depending on the strength of the field. This also implies that for UHECRs to emerge from the vicinity of a neutron star, they should be accelerated several hundred radii from the star to avoid synchrotron losses. Moreover, synchrotron radiation directly affects the relevance of other loss mechanisms such as curvature radiation and trident pair production; curvature radiation is significant only if synchrotron radiation forces particles to follow magnetic field lines, and energy losses from trident pair production are much less than those due to synchrotron radiation, in our range of ion energies and magnetic field strengths (see, e.g., Erber 1966).

Our final point in this section is that even if the magnetic field is too weak to induce energy losses, e.g. for propagation beyond $r_{L}$, it may still significantly affect the trajectories of cosmic rays. This will affect low-energy particles more than high-energy particles and, at a given energy, will delay the emergence of heavier nuclei compared to protons. This may lead to a filter that selectively allows higher energy particles to escape, and which affects the observed composition of particles as a function of their energy.

\subsubsection{Synchrotron Radiation}

A particle of charge $q$ and mass $m$, with associated Lorentz factor $\gamma$ and energy $E$, propagating at velocity $\beta c$ at an angle $\alpha$ with respect to a magnetic field of strength $B$, loses energy to synchrotron radiation at a chracteristic rate

$$
\frac{\dot{E}}{E}=-\frac{2}{3} r_{0}^{2} B^{2} \gamma \beta^{2} \frac{\sin ^{2} \alpha}{m c},
$$

where $r_{0}=q^{2} / m c^{2}$ is the classical radius of the particle. Thus, a nucleus of charge $Z e$ and mass $A m_{p}$ has a loss rate of

$$
\frac{\dot{E}}{E}=3 \times 10^{5} \frac{Z^{4}}{A^{3}} \gamma \beta^{2} B_{12}^{2} \sin ^{2} \alpha \mathrm{s}^{-1} .
$$

Note that for a fixed energy $E, \gamma \sim 1 / m$ and $\dot{E} / E \sim m^{-4}$.

At a qualitative level, synchrotron losses are unimportant at a radius $r$ from the neutron star when the characteristic energy loss timescale at that radius exceeds the time to propagate a distance $r$, if the cosmic ray is traveling in a straight line away from the surface. Assuming a dipolar magnetic field of strength $B_{0}$ at the poles, if $\sin \alpha=1$, then protons or iron nuclei with $\gamma=10^{9}$ suffer negligible synchro- 
tron losses for $B_{12} \simeq 10^{-6}$, which occurs at $r \simeq 10^{8} \mathrm{~cm}$ for a typical surface field $B_{0}=10^{12} \mathrm{G}$. The very strong dependence of the loss rate on distance $\left(\sim r^{-6}\right.$, for a dipole field) implies a transition radius $r \sim 10^{8} \mathrm{~cm}$ between regions of significant and insignificant synchrotron losses, for straightline propagation. In reality, the trajectories of cosmic rays may be deflected by the magnetic field, which increases the path length, but, given the steep dependence of the loss rate on radius, we expect that for $r \gtrsim 10^{9} \mathrm{~cm}$ the loss is insignificant. Thus, $r \gtrsim 10^{9} \mathrm{~cm}$, corresponding to acceleration at an outer gap or at the light cylinder (for $P \gtrsim 0.2 \mathrm{~s}$ ), is a promising location for surviving UHECRs.

\subsubsection{Curvature Radiation}

Consider now the $r \lesssim 10^{9} \mathrm{~cm}$ region, where particles follow field lines. The most important energy loss process is then curvature radiation (see, e.g., Sorrell 1987). The power in curvature radiation for a nucleus of charge $Z$ and Lorentz factor $\gamma$ moving along field lines with radius of curvature $R$ is

$$
P=\frac{2}{3} \frac{(Z e)^{2} c}{R^{2}} \gamma^{4}
$$

If the power source is a constant electric field of magnitude $\mathscr{E}=10^{12} \mathscr{E}_{12} \mathrm{~V} \mathrm{~cm}^{-1}$, then the energy at which the losses in curvature radiation equal the power gain through the electric field is

$$
E_{\max } \approx 10^{18}\left(\frac{A}{56}\right)\left(\frac{Z}{26}\right)^{-1 / 4} R_{6}^{1 / 2} \mathscr{E}_{12}^{1 / 4} \mathrm{eV} .
$$

As expected, the maximum energy rises with increased radius of curvature. The radius of curvature at the stellar radius $R_{*} \approx 10^{6} \mathrm{~cm}$ of a dipole field line of maximum radius $R_{\max } \gg R_{*}$ is

$$
R \approx 2\left(R_{\max } R_{*}\right)^{1 / 2} .
$$

Thus, since the area of the polar cap is proportional to $R_{\max }^{-1}$, the angular deviation of a field line from the magnetic pole that just barely allows particles of energy greater than $E_{\max }$ to escape scales as $\theta \sim E_{\max }^{-2}$. If particles are injected uniformly along the polar cap, this implies a spectrum proportional to $E^{-5}$ with a sharp cutoff at the maximum potential drop. If the original source of energetic particles is at the neutron star surface, this implies that either particle injection is strongly biased toward the magnetic pole or there are further acceleration mechanisms that harden the spectrum.

\subsubsection{Propagation outside the light cylinder}

Outside the light cylinder, conservation of magnetic flux implies that the magnetic field scales as $B \sim r^{-1}$. Since the radial component of the magnetic field scales as $B_{r} \sim r^{-2}$ far from the light cylinder, the azimuthal component dominates. Therefore, most nuclei traveling away from the star must eventually cross field lines. If the gyration radius of a particle $r_{g}$ at the light cylinder is less than $r_{L}$, the particle might become trapped. This may lead to collisionless shocks or other mechanisms by which energy can be redistributed amongst the particles. Note that since

$$
\frac{r_{g}}{r_{L}}=0.03 E_{15} B_{0,12}^{-1}\left(\frac{Z}{26}\right)^{-1} P_{-1}^{2},
$$

this can also act as a filter which preferentially allows higher energy particles to escape. Note also that for a given energy of the particle, low $-Z$ species escape sooner than high- $Z$ species. For example, iron nuclei of energy $10^{15} \mathrm{eV}$ are trapped only up to a neutron star period of $P \sim 0.6 \mathrm{~s}$, whereas protons escape for periods greater than about $0.1 \mathrm{~s}$. Alternatively, for a given neutron star period or stage in the star's lifetime, protons need less energy to overcome this effect than do iron nuclei.

\section{CONCLUSIONS}

In this paper, we have examined the case for generating UHECRs from isolated neutron stars. From overall power and energy loss criteria, we have strongly constrained possible models. We find that if isolated neutron stars produce most UHECRs, the generation mechanism must be ultimately powered by rotation and the particles must come from near the star rather than from the interstellar medium. We also find that potential drops along magnetic fields cannot accelerate particles to energies above $10^{15} \mathrm{eV}$, since electron-positron pair cascades are created that significantly reduce the available energies. Unless the acceleration takes place farther than $\sim 10^{9} \mathrm{~cm}$ from the star, synchrotron losses are likely to dissipate a significant fraction of the particle energy.

High-energy gamma rays from pulsars also provide evidence against a substantial fraction of the rotational energy of neutron stars being converted to particles of Lorentz factor $\gtrsim 10^{7}$ within the light cylinder. These objects have a gamma-ray luminosity that is only a small fraction of their total spin-down power, typically less than $10 \%$ ( Nel et al. 1996, and references therein). This is contrary to what would be observed if energetic particles were accelerated close to rapidly rotating, strongly magnetized neutron stars (as we discuss in § 4). Thus, if UHECRs come from isolated neutron stars, the acceleration region must be near or outside $r_{L}$. Finally, we note that if acceleration does indeed take place beyond the light cylinder, the azimuthal nature and radial dependence of the magnetic field in this region may form a magnetic bottle that preferentially lets out higher energy particles.

We thank M. Ruderman for pointing out the relevance of multiwavelength observations of pulsars. We also thank G. Sigl, R. Epstein, and K. Green for useful references and discussions. A. V. and A. O. were supported in part by the DOE through grant DE-FG0291 ER40606. M. C. M. gratefully acknowledges the support of a Compton GRO Fellowship, and NASA grants NAG 5-2868 and 5-2687.
Axford, W. I. 1994, ApJS, 90, 937

Becker, R. H., \& Helfand, D. J. 1983, Nature, 302, 688

Biermann, P. L. 1993, A\&A, 271, 649

Bildsten, L., Salpeter, E. E., \& Wasserman, I. 1992, ApJ, 384, 143

Bird, D. J., et al. 1994, ApJ, 424, 491

Blaes, O., \& Madau, P. 1993, ApJ, 403, 690 (BM93)

Blaes, O., Warren, O., \& Madau, P. 1995, ApJ, 454, 370

\section{REFERENCES}

Bondi, H., \& Hoyle, F. 1944, MNRAS, 104, 273

Cheng, K. S., Ho, C., Ruderman, M. A. 1986, ApJ, 300, 500

Erber, T. 1966, Rev. Mod. Phys., 38, 626

Frail, D. A., Goss, W. M., \& Whiteoak, J. B. Z. 1994, ApJ, 437, 781

Gaisser, T. K., et al. 1993, Phys. Rev. D, 47, 1919

Harding, A. K. 1990, Nucl. Phys. B (Proc. Supp.), 14A, 3

Illarionov, A., \& Sunyaev, R. 1975, A\&A, 39, 185 
Lyne, A. G., \& Lorimer, D. R. 1994, Nature, 369, 127

Madau, P., \& Blaes, O. 1994, ApJ, 423, 748 (MB94)

Milgrom, M., \& Usov, V. 1996, Astropart. Phys., 4, 365

Miller, M. C., \& Neuhauser, D. 1991, MNRAS, 253, 107

Müller, D., et al. 1991, ApJ, 374, 356

Nel, H. I., et al. 1996, ApJ, 465, 898

Rösner, W., Wunner, G., Herold, H., \& Ruder, H. 1984, J. Phys. B, 17, 29
Ruderman, M. A., \& Sutherland, P. G. 1975, ApJ, 196, 51

Shapiro, S. L., \& Teukolsky, S. A. 1983, Black Holes, White Dwarfs, and Neutron Stars (New York: Wiley), 278

Shemi, A. 1995, MNRAS, 275, 115

Sorrell, W. H. 1987, ApJ, 323, 647

Timmes, F. X., Woosley, S. E., \& Weaver, T. A. 1996, ApJ, 457, 834

Vietri, M. 1996, MNRAS, 278, L1 